\title{
Perspectivas y desafíos de los fonoaudiólogos en la actividad académica en Chile: una descripción preliminar
}

\author{
Perspectives and challenges of speech and language \\ therapists in academic activity in Chile: A preliminary \\ description
}

Manuel del Campo

Escuela de Fonoaudiología, Universidad Santo Tomás

\section{Angélica Silva-Ríos}

Escuela de Fonoaudiología, Universidad Santo Tomás

Jorge Valdés

Universidad Santo Tomás

\section{Contacto con el autor: Manuel del Campo \\ Viña del Mar - Chile Correo-e:mdelcampo2@santotomas.cl}

Recibido: 30/01/2019 Aceptado: 03/06/2019

\section{RESUMEN}

El fonoaudiólogo en Chile es el profesional encargado de la evaluación, diagnóstico, rehabilitación, promoción y prevención de los trastornos del lenguaje, el habla, la deglución, la audición, la voz y la comunicación. Actualmente, 29 casas de estudio superior dictan la carrera de Fonoaudiología y otorgan el título profesional de Fonoaudiólogo en Chile. Dada la evolución de la profesión, es necesario que se reflexione respecto de su quehacer formador. Los objetivos del presente trabajo son: a) Describir las características de los fonoaudiólogos que se dedican a la academia en diferentes universidades chilenas y b) exponer los aspectos de autorreflexión de la formación de pregrado y formación continua en Fonoaudiología. Para ello, se realizó un estudio observacional descriptivo mediante la aplicación de una encuesta estructurada a 33 académicos representantes de 12 universidades chilenas. Los resultados mostraron que el $60,6 \%$ de los académicos asistentes ostenta el grado de Magíster. Un 59,4\% de los docentes tienen una dedicación laboral completa, centrando su carga en la gestión o la docencia. Sólo un $12,1 \%$ de los informantes reporta el desarrollo de actividades vinculadas con la investigación. El 87,9\% de los consultados refiere que la formación de pregrado en Chile es de calidad y un $57,6 \%$ considera que la oferta nacional de posgrado no es pertinente para el desarrollo de la profesión. Según estos antecedentes, se discuten aspectos relacionados con: afiliación, actividades académicas, distribución de carga laboral, formación en el pregrado y formación continua en Fonoaudiología.

Palabras clave: fonoaudiología, educación superior, educación.

\section{ABSTRACT}

In Chile, Speech and Language therapists (SLT) are the professionals in charge of topics as diverse as evaluation, diagnosis, rehabilitation and health promotion of language disorders, speech, swallowing, hearing, voice, and communication. Currently, 29 higher education institutions include majors in SLT in Chile. Given the evolution of the profession, some reflections and considerations on the practices of SLT training are in order. The objective of this study was to describe some aspects related with the characteristics of speech therapists in academic activity in Chile and present a discussion about practices of SLT training. A descriptive observational study was conducted by applying a structured survey to 33 academic representatives of 12 Chilean universities. The results showed that $60.6 \%$ of the attending academics hold the Master's degree. A $59.4 \%$ of the teachers consulted have a complete work dedication, focusing their load on management or teaching. Only $12.1 \%$ of the informants report the development of activities related to research. An $87.9 \%$, of respondents say that undergraduate training in Chile is of quality and $57.6 \%$ consider that the national postgraduate offer is not useful for the development of the profession. According to this background, aspects related to: affiliation, academic activities, workload distribution, undergraduate training and continuing training in speech therapy are discussed.

Keywords: speech and language therapy, higher education, education. 


\section{Introducción}

En Chile el fonoaudiólogo es el profesional encargado de la evaluación, diagnóstico, rehabilitación, promoción y prevención de los trastornos del lenguaje, del habla, la deglución, la audición, la voz y la comunicación (Martínez et al., 2006).

La Fonoaudiología ha experimentado, en las últimas décadas, una suerte de transformación que la ha llevado a ser concebida como la disciplina que se ocupa del bienestar comunicativo de las personas (Romero, 2012). Según ASHA (2016), el alcance de la práctica se amplía concibiendo el bienestar como las acciones y actitudes que mejoran la calidad de vida de las personas. Así, incorpora en su visión, la posibilidad de intervenir desde la promoción y la prevención de factores de riesgo en la salud comunicativa de la comunidad.

Desde la apertura de la primera Escuela de Fonoaudiología, en la Universidad de Chile hacia el año 1955 (Martínez et al., 2006), hasta la fecha se ha producido un crecimiento en el desarrollo, lo que ha complejizado. Lo anterior se refleja en la formación de diversas organizaciones relacionadas con la disciplina. Las más relevante son: el Colegio de Fonoaudiólogos de Chile A.G. creado en 1983 (Colegio de Fonoaudiología, s. f.), la Asociación de Estudiantes de Fonoaudiología de Chile (Aefoch) conformada en 1994, la Organización de Fonoaudiólogos para Chile, FOPACH (2016) constituida el año 2016, la Asociación Científica Chilena de Fonoaudiología (ASOCHIFA), Sociedad Chilena de Fonoaudiología (Sochifo) y la Asociación Gremial de Fonoaudiólogos de la región de Aysén (AGFONAY) que se constituyó el año 2017.
Actualmente, en el área de la salud los profesionales fonoaudiólogos tienen una escasa participación en la atención primaria (Martínez et al., 2006). Ello debido, en parte, a las políticas públicas que no incorporan a este profesional dentro de sus equipos de trabajo. Al respecto, cabe destacar la iniciativa enmarcada dentro del modelo biopsicosocial de salud, la Rehabilitación Basada en la Comunidad (RBC), orientada a fortalecer la Atención Primaria en Salud (APS) (Ossandón, 2014). Esta iniciativa demanda al fonoaudiólogo competencias que le permitan planear y ejecutar acciones con una clara perspectiva comunitaria. Dichas competencias deben lograrse dentro de la formación de pregrado.

Actualmente, 29 casas de estudio superior dictan la carrera de Fonoaudiología (Ministerio de Educación, 2018) en horario diurno o vespertino y otorgan el título profesional de fonoaudiólogo, con su respectiva licenciatura. Además, el Instituto Profesional de Chile también entrega el título de fonoaudiólogo (Instituto Profesional de Chile, 2018).

El año 2017 se matricularon 2.513 estudiantes a nivel nacional (Ministerio de Educación, 2017), contando con un número total de 11.799 educandos en aula. El número de titulados durante el año 2016 fue de 1.623 sujetos, con una duración de la carrera de al menos 12 semestres.

Desde un punto de vista laboral, cabe mencionar que la empleabilidad al primer año de egreso alcanza un $61,9 \%$, elevándose a un $83,6 \%$ al segundo año de titulación (Ministerio de Educación, 2017, 2018). La profesión en Chile cuenta adicionalmente con instrumentos de difusión de la disciplina, como la Revista Chilena de Fonoaudiología (Departamento de Fonoaudiología, 2018) y la Revista Chilena de Ciencias 
de la Rehabilitación en Salud, cuyo editor principal es fonoaudiólogo (Universidad del Bio-Bio, 2018).

Dentro de los ámbitos clásicos de la práctica fonoaudiológica, ha de mencionarse que, a nivel educativo, "si bien se han generado numerosas plazas de trabajo, ya sea en Escuelas Especiales de Lenguaje o Programas de Integración Escolar, no existe una declaración clara respecto del rol que el fonoaudiólogo debe cumplir en dicho ámbito" (Torres, Vega, y Del Campo, 2015). De acuerdo con esto, Torres et al. (2015) comunican que a pesar de la claridad existente en relación con el desafío que implica la inclusión escolar de niños con necesidades educativas especiales, en Chile existe una brecha conceptual y procedimental entre la práctica fonoaudiológica observada en educación y la declarada por el Ministerio de Educación.

En este devenir han surgido reflexiones disciplinares cuyo acuerdo ha generado un claustro anual convocado por la Universidad San Sebastián (Universidad San Sebastián, 2018), denominado Jornada Nacional de Académicos de Fonoaudiología. En este espacio se reúnen los directivos y académico de las escuelas formadoras a nivel nacional. El año 2017, se celebró la V jornada se celebró en la ciudad de Viña del Mar, sirviendo como anfitriona la Escuela de Fonoaudiología, de la Facultad de Salud, de la Universidad Santo Tomás (Universidad Santo Tomás, 2018). En este encuentro se ofrecieron diversas exposiciones y mesas de trabajo en torno al tema "La enseñanza de la Fonoaudiología en la Educación Superior: Perspectivas y Desafíos de la Fonoaudiología, una visión desde la Academia".

Dada la evolución de la formación de fonoaudiólogos en Chile y los diferentes requerimientos que la sociedad impone a estos profesionales se plantea la siguiente pregunta de investigación: ¿Cuáles son las características y los desafíos de los fonoaudiólogos en la actividad académica en Chile? Por su parte los objetivos fueron: a) Describir las características de los fonoaudiólogos que se dedican a la academia en las diferentes universidades chilenas y b) exponer los aspectos de autorreflexión de la formación de pregrado y formación continua en Fonoaudiología. Para ello, se consideran tres dimensiones: a) Afiliación, actividades académicas y distribución de carga laboral, b) autorreflexión de la formación en el pregrado y c) formación continua.

\section{Metodología}

\section{Participantes}

El universo estuvo compuesto por fonoaudiólogos y fonoaudiólogas, chilenos(as) y extranjeros(as) asistentes a la V Jornada de Académicos en Fonoaudiología. Se realizó un muestreo no probabilístico e intencionado. La muestra estuvo conformada por 32 fonoaudiólogos(as) y un psicólogo(a).

\section{Aspectos éticos}

La presente investigación adscribe a la declaración de Helsinki y los participantes no fueron expuestos a procedimientos que pusieran en riesgo su integridad física o emocional. Al inicio de la encuesta se informó a los consultados respecto de la estructura y fines de la encuesta. Los profesionales que optaron por participar confirmaron su colaboración mediante un 
consentimiento informado digital. Las identidades de los participantes fueron codificadas en formato numérico, datos que se encuentran alojados en el computador personal del investigador principal.

\section{Instrumento}

Para recopilar la información se diseñó una encuesta en formato digital (Google Forms), que explora 3 dimensiones: a) Afiliación, actividades académicas y distribución de carga laboral, b) Autorreflexión de la formación en el pregrado y c) formación continúa en Fonoaudiología. La encuesta fue sometida a validación de contenido por parte de tres fonoaudiólogos independientes, quienes obtuvieron $100 \%$ de coincidencia en los reactivos presentados.

Las respuestas de los participantes fueron tabuladas en una planilla de Excel para luego ser procesada en el Software de análisis estadístico Stata 14. Se utilizó estadística descriptiva y se graficaron los hallazgos más relevantes.

\section{Resultados}

Los principales resultados se presentarán de acuerdo con las dimensiones planteadas, con objeto de facilitar la lectura.

\section{a) Afiliación, actividades académicas y distribución de carga laboral}

El $97 \%$ de los encuestados son fonoaudiólogos, sólo uno de los consultados tiene formación de pregrado en psicología.

El $60,6 \%$ de los encuestados tiene grado de Magíster, el 30,3\% de licenciado y sólo el 6,1\% tiene estudios de tercer ciclo (Ph.D).

El Gráfico 1 muestra que en las universidades Santo Tomás (36.4\%), Andrés Bello (15.2\%) y Católica Silva Henríquez (12.1\%) se concentra la mayoría de los académicos encuestados.

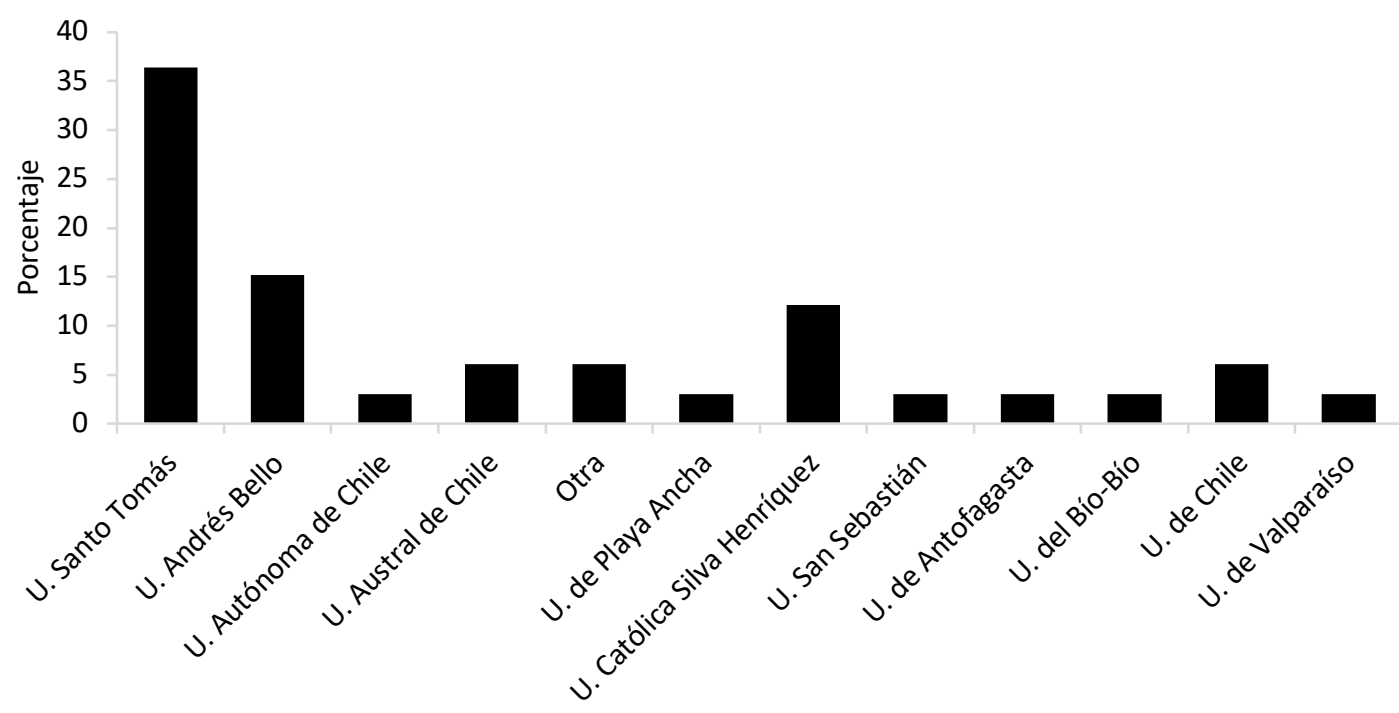

Gráfico 1. Universidad de origen de los académicos. 
El $87,9 \%$ de los académicos asistentes tiene contrato de planta en sus respectivas universidades y sólo un $12,1 \%$ es profesor a honorarios.

Un $58 \%$ de los docentes tiene una dedicación laboral completa vinculada a sus universidades. Por otra parte, el $30 \%$ cuenta con un contrato de media jornada. Sólo un profesor (3,3\%) informa tener contrato menor a 12 horas. El gráfico 2 ilustra los datos comentados.

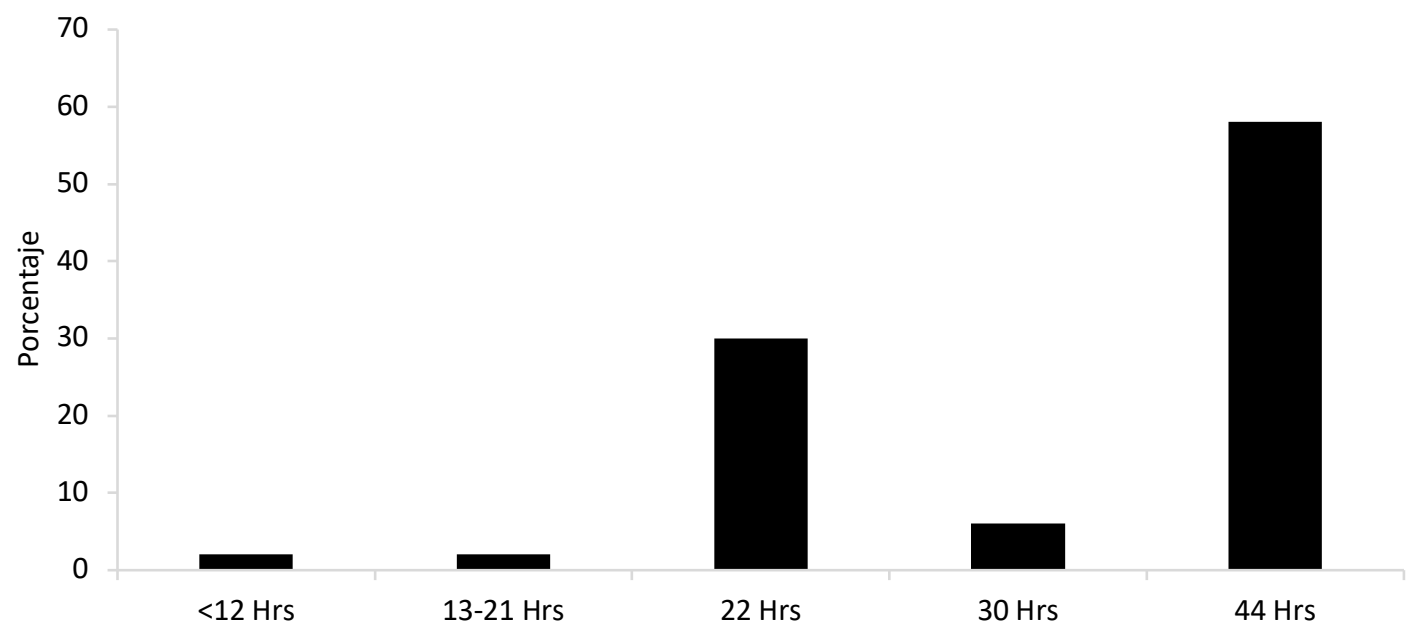

Gráfico 2. Dedicación horaria semanal.

En el Gráfico 3 se observa que las principales actividades académicas se concentran en la gestión $(42,4 \%)$, seguido de docencia $(36,4 \%)$. Finalmente, la investigación y la tutoría clínica son las actividades que realiza un menor porcentaje de académicos (12,1\% 9. 5\% respectivamente).
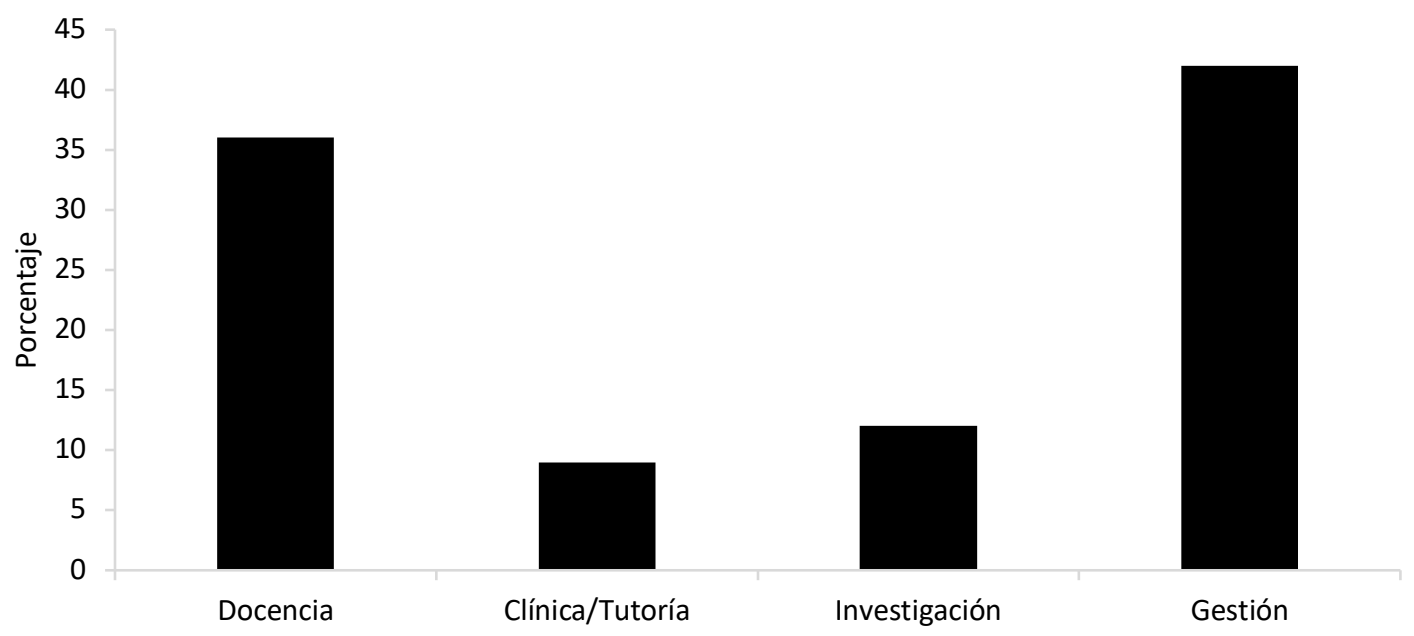

Gráfico 3. Principales actividades docentes. 
Los docentes encuestados afirman que conocen el modelo educativo de la casa de estudio donde se desempeñan y consideran que es pertinente para la formación fonoaudiológica actual. Adicionalmente, un $84,8 \%$ declara que su dedicación horaria les permite cumplir de manera efectiva sus responsabilidades.

\section{b) Autorreflexión de la formación en el pregrado}

Un $87,9 \%$ de los docentes considera que la formación de pregrado en Chile (independiente de la universidad donde se desempeñan) es de calidad. En tanto el $12.1 \%$ de académicos creen que la calidad de formación de pregrado es deficiente.

Por otra parte, un $45 \%$ piensa que la actual formación de pregrado no está alineada con los retos que enfrentarán los profesionales jóvenes al ingresar al mundo laboral.

El $42.4 \%$ de los académicos consideran que los principales desafíos de la formación de pregrado de los fonoaudiólogos apuntan a fortalecer la práctica basada en la evidencia. A su vez, el $21.2 \%$ de ellos piensa que se debe alcanzar una madurez gremial. Finalmente, un $15.2 \%$ afirma que es necesario incorporar modelos de intervención que se ajusten a los actuales hallazgos científicos. Los datos están expuestos en el Gráfico 4.

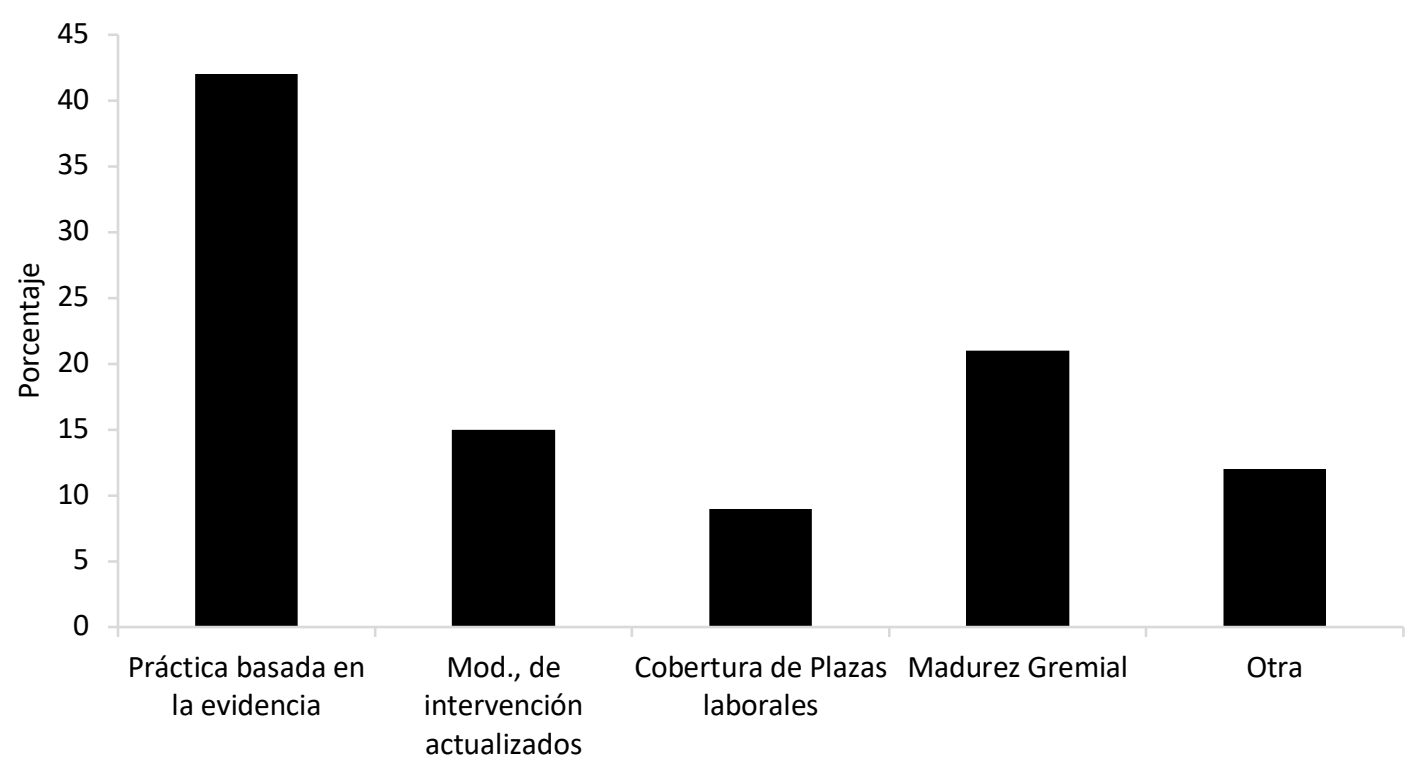

Gráfico 4. Principales desafíos declarados por académicos fonoaudiólogos en Chile.

Un $39,4 \%$ de los académicos informan que sus estudiantes de pregrado realizan actividades de pasantía o intercambio internacional.

\section{c) Formación continua en Fonoaudiología}

El $57,6 \%$ de los académicos refiere que la oferta nacional de posgrado no es pertinente para el desarrollo de la profesión, mientras que un $42,4 \%$ considera que existe una oferta de programas que 
posibilitan el desarrollo profesional.

El Gráfico 5 muestra los datos sobre la valoración que tienen los académicos de la formación continua en la disciplina. Un $48 \%$ de los docentes informan que optarían por cursar un programa de doctorado y un $36 \%$ preferiría un programa de magíster y un $12 \%$ accedería a la formación de especialista.

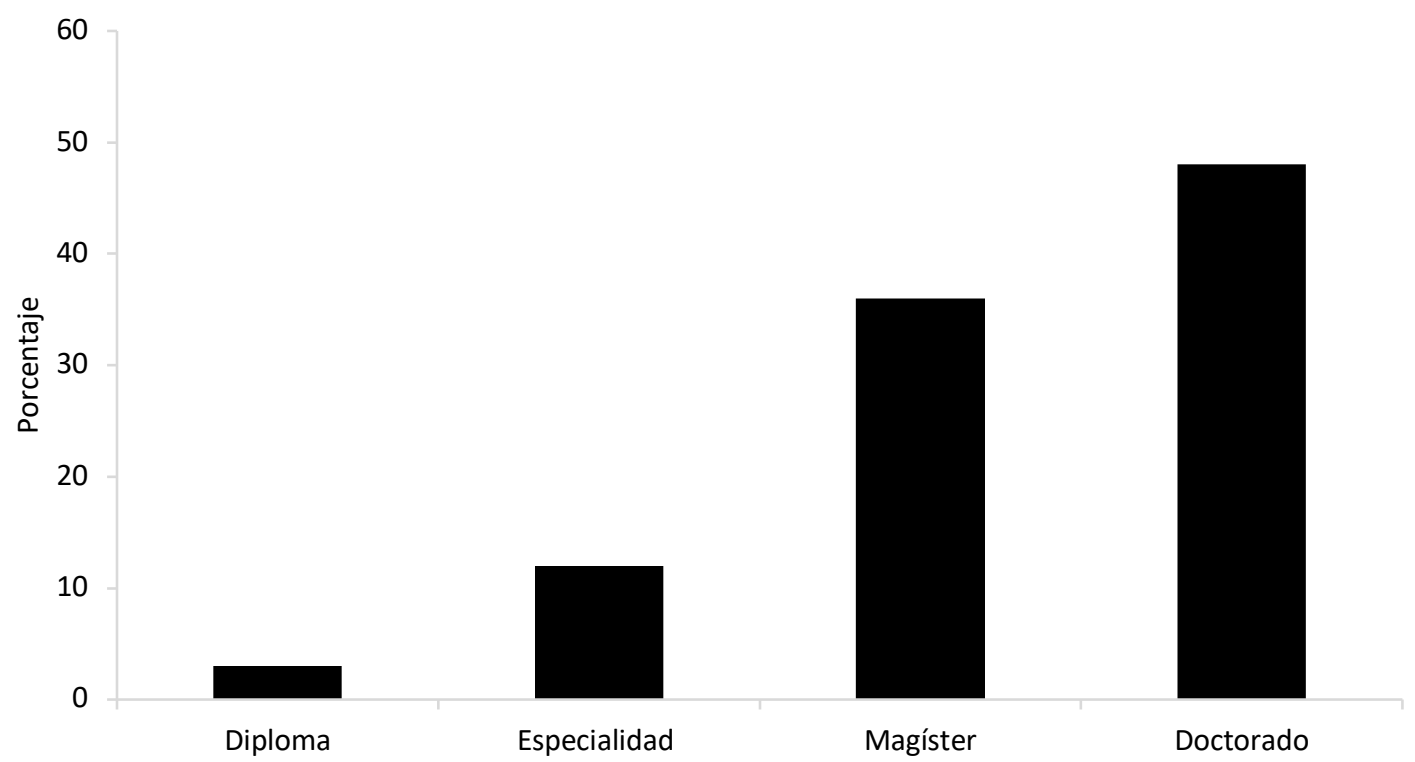

Gráfico 5. Tipo de programa de postgrado de interés.

Un 60,6\% de los fonoaudiólogos docentes optarían por dedicar tres días mensuales (seguidos) a su formación continua. Un 15,2\% desearían cuatro sesiones mensuales distribuidas en un día semanal en formato diurno, o bien tres sesiones semanales en jornada vespertina 15,2\%. Sólo el 9.1\% de académicos declara estar disponible para optar por la dedicación exclusiva.
El Gráfico 6 informa que, del total de las menciones, el $17,7 \%$ refiere la necesidad de fortalecer la formación en áreas como la comunicación social a nivel de posgrado, un $16,6 \%$ en cognición y un $12,5 \%$ en deglución. Por otra parte, un disminuido porcentaje plantea que se debe orientar la formación del posgrado hacia áreas como la resonancia 1,1\%, la estética facial $4,2 \%$ o audiología $4,2 \%$. 


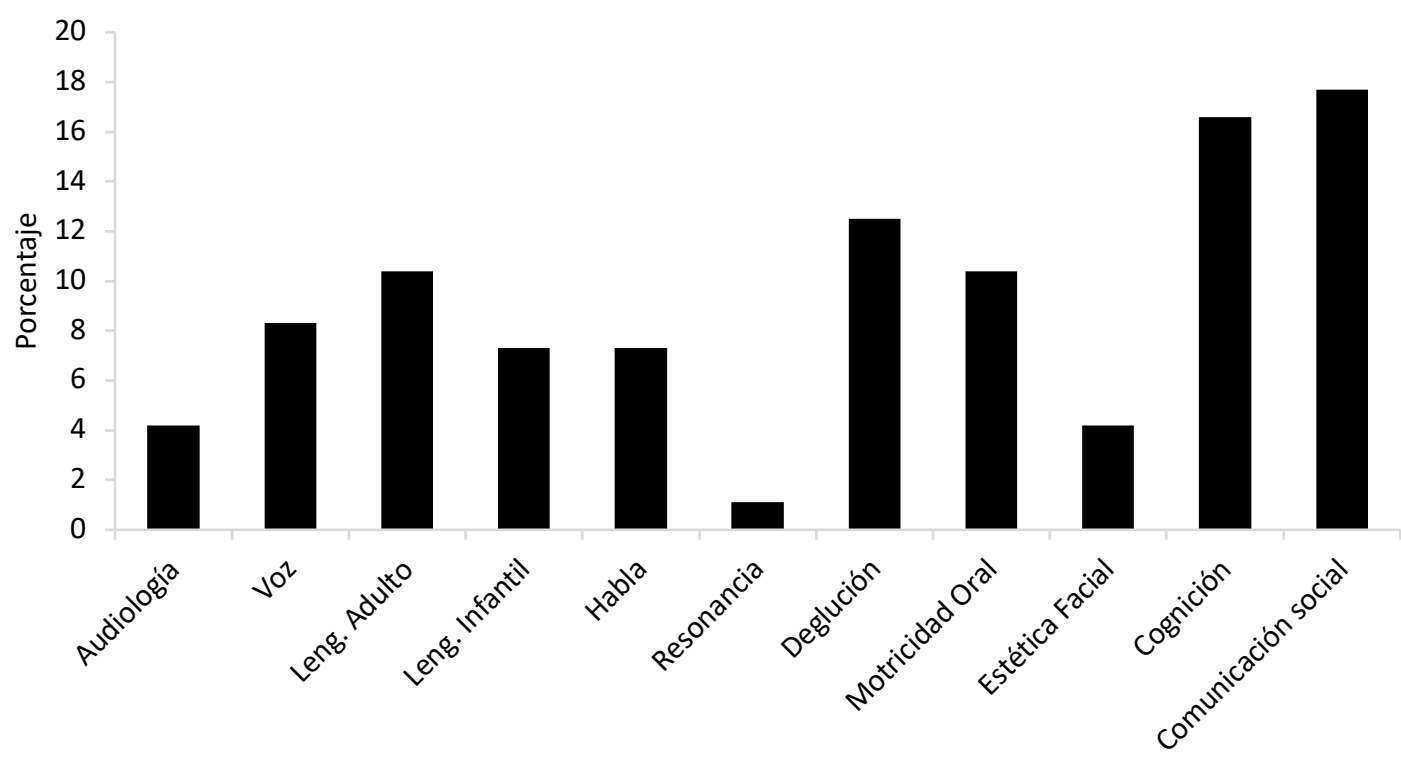

Gráfico 6. Áreas a fortalecer en la formación de postgrado.

\section{Discusión}

Tras examinar aspectos fundamentales que conciernen al quehacer de los fonoaudiólogos dedicados a la actividad académica en Chile, surgen algunas reflexiones sobre la disciplina, desde las necesidades sociosanitarias del país (Medina y Kaempffer, 2007) y desde mundo laboral.

Las reflexiones que se expondrán se desarrollarán a partir de los tópicos más relevantes analizados en esta investigación.

\section{Afiliación, actividades académicas y distribución de carga laboral.}

Evidencia previa muestra que la contratación de investigadores (Hernández-Jaramillo, Cruz-Velandia, y Torres-Narváez, 2010) debiera generar nuevo conocimiento (Pava-Ripoll, Payán, y Reyes, 2011). Ello puede contribuir a la utilización de la práctica basada en la evidencia. En Chile existe un escaso cuerpo de académicos fonoaudiólogos con grado de doctor. Tampoco se cuenta con suficientes equipos que se dediquen de manera formal a la investigación y/o innovación. Lo anterior se ve reflejado en el presente estudio, ya que, si bien un $58 \%$ de los académicos informantes en este estudio cuentan con jornada laboral completa, sus funciones se concentran en labores relacionadas con la gestión y no con actividades relacionadas con la creación de nuevo conocimiento (Gráfico 2).

\section{Formación en el pregrado}

Las universidades deben entregar herramientas actitudinales, cognitivas y procedimentales (Matus et al., 2017) desde el pregrado a sus educandos con objeto de que estos puedan enfrentar, de manera exitosa, los desafíos del mundo laboral. Lo anterior requiere evidentemente de la construcción de una matriz conceptual (Hawes et al., 2017) que colabore no tan sólo con la adquisición de conocimientos, sino 
con la formación integral de ciudadanos que contribuyan a mejorar la forma de comunicar y habitar de las personas con las cuales se relacionan profesionalmente.

Tanto la creación de conocimiento derivada de la asociatividad interuniversitaria como el desarrollo de destrezas transversales desde el pregrado, podrían verse fortalecidas a través del establecimiento de vínculos internacionales (Brunner y Pedraja-Rejas, 2017), que permitan la ejecución de pasantías, jornadas de investigación e intercambio. En este contexto, los profesionales dedicados a la docencia consideran que uno de los principales desafíos de la disciplina se refiere al fortalecimiento de la práctica basada en la evidencia (gráfico 4).

\section{Formación continua en Fonoaudiología}

Los profesionales plantean la necesidad de ampliar la oferta de posgrado hacia el tercer ciclo. Es decir, surge la necesidad de contar con ofertas a nivel nacional de programas de doctorado (Gráfico 5), siendo las áreas de comunicación social, cognición y deglución (Gráfico 6) las que exigen mayor fortalecimiento, lo que parece estar en concordancia con la experiencia internacional (Lizarazo-Camacho y Pereira, 2015).

Es necesario analizar de manera constante el quehacer fonoaudiológico en la actividad académica, ya que los lineamientos entregados por las casas de estudio superior impactarán de manera consistente en el aporte que los egresados realicen a la sociedad. De igual modo, es necesario que el análisis preliminar reportado por esta investigación pueda completarse mejorando el instrumento para la obtención de los datos. Además, se debe ampliar la muestra de estudio con el objeto de conocer de forma más exhaustiva aspectos disciplinares y formativos de la formación universitaria en Fonoaudiología.

Los antecedentes presentados invitan no tan sólo a la universidad (Torres et al., 2015), sino también al gremio profesional a replantear cursos de acción, con el fin último de colaborar en la mejora de las condiciones basales del pre y posgrado. Así, se puede contribuir a una formación de excelencia en Fonoaudiología, el desarrollo de un cuerpo profesional de alta calidad, que apoye de forma objetiva al bienestar comunicativo de la sociedad.

\section{Referencias}

American Speech-Language-Hearing Association, (ASHA). (2016). Scope of Practice in Speech-Language Pathology. https://doi.org/10.1044/policy.SP2016-00343

Brunner, J., \& Pedraja-Rejas, L. (2017). Challenges to higher education governance in Ibero-America. Ingeniare. Revista chilena de ingeniería, 25(1), 2-7. https://doi.org/10.4067/S0718-33052017000100002

Colegio de Fonoaudiología, (COLFONO). (s. f.). Colegio de Fonoaudiólogos de Chile A.G. - Sections. Recuperado 18 de marzo de 2018, de Historia website: http://www.colfonochile.cl/historia

Departamento de Fonoaudiología. (2018). Revista Chilena de Fonoaudiología [Página institucional]. Recuperado 18 de marzo de 2018, de Revista Chilena de Fonoaudiología, Universidad de Chile website: https://revfono.uchile.cl/

Hawes, G., Rojas-Serey, A. M., Espinoza, M., Oyarzo, S., Castillo-Parra, S., Castillo, M., \& Romero, L. (2017). Desarrollo de una matriz conceptual para la innovación curricular en profesiones de la salud. Revista Médica de Chile, 145(9), 1193-1197. https://doi.org/10.4067/s003498872017000901193

Hernández-Jaramillo, J., Cruz-Velandia, I., \& Torres-Narváez, M. (2010). Investigación clínicas en fonoaudiología: Análisis de la literatura científica 2005-2009. Revista de la Facultad de Medicina, 58(3), 204-213. Recuperado de 
https://revistas.unal.edu.co/index.php/revfacmed/article/ view/18573

Instituto Profesional de Chile, (IPChile). (2018). Fonoaudiología [Página institucional]. Recuperado 18 de marzo de 2018, de IPChile website: https://www.ipchile.cl/carreras/fonoaudiologia/

Lizarazo-Camacho, A. M., \& Pereira, O. (2015). Caracterización de la proyección social del programa de fonoaudiología de la Corporación Universitaria Iberoamericana. Areté, 15(1), 19-38. Recuperado de https://arete.ibero.edu.co/article/view/922

Martínez, L., Cabezas, C., Labra, M., Hernández, R., Martínez, L. M., Cerutti, M., \& Malebrán, C. (2006). La logopedia en Iberoamérica. Actas del XXV Congreso de Logopedia, Foniatría y Audiología, 14. Recuperado de http://www.sld.cu/galerias/pdf/sitios/rehabilitacion-

logo/historia_en_iberoamerica.pdf

Matus, O., Ortega, J., Parra, P., Ortiz, L., Márquez, C., Stotz, M., ... Fasce, E. (2017). The educational context to train health care professionals. A qualitative approach. Revista Médica de Chile, 145(7), 926-933. https://doi.org/10.4067/s0034-98872017000700926

Medina, E., \& Kaempffer, A. M. (2007). The Health Careers teaching system in Chile. Revista Médica de Chile, 135(10), 1346-1354. https://doi.org/10.4067/S003498872007001000018

Ministerio de Educación, (MINEDUC). (2017). InicioEducación Superior [Página gubernamental de Chile]. Recuperado 18 de marzo de 2018, de Subsecretaría de Educación Superior website: https://educacionsuperior.mineduc.cl/

Ministerio de Educación, (MINEDUC). (2018). MI FUTURO [Página gubernamental de Chile]. Recuperado 18 de marzo de 2018, de Mineduc website: https://www.mifuturo.cl/

Organización de Fonoaudiólogos para Chile, (FOPACH). (2018). Sobre nosotros-Fopach. Recuperado 18 de marzo de 2018, de Fopach website: https://fopach.cl/sobrenosotros/

Ossandón, M. P. (2014). Rehabilitación basada en la comunidad frente a la realidad chilena. Revista Chilena de Terapia Ocupacional, 14(2), 219-230. https://doi.org/10.5354/0719-5346.2014.35724

Pava-Ripoll, N., Payán, C., \& Reyes, A. (2011). Aportes desde la investigación formativa a la producción en Fonoaudiología: El caso de una universidad colombiana. Revista da Sociedade Brasileira de Fonoaudiologia, 16(4), 377-383. https://doi.org/10.1590/S151680342011000400003

Romero, L. (2012). Desplazamiento respecto de los saberes disciplinares de la Fonoaudiología. Revista Chilena de Fonoaudiología, 11, 99-106. https://doi.org/10.5354/07194692.2012.24520

Torres, A., Vega, Y., \& Del Campo, M. (2015). Autorreflexión sobre el desempeño profesional del fonoaudiólogo(a) en el sector educativo: Una aproximación a la construcción del rol. Revista Chilena de Fonoaudiología, 14, 103-117. https://doi.org/10.5354/rcdf.v14i0.37738

Universidad del Bio-Bio. (2018). Revista Chilena de Ciencias de Rehabilitación en Salud [Página institucional]. Recuperado 18 de marzo de 2018, de Revista Chilena de Ciencias de Rehabilitación en Salud website: http://revistas.ubiobio.cl/index.php/CRS

Universidad San Sebastián, (USS). (2018). Malla curricular [Página institucional]. Recuperado 18 de marzo de 2018, de Facultad de Ciencias de la Salud website: http://www.uss.cl/ciencias-

salud/carrera/fonoaudiologia/malla-curricular/

Universidad Santo Tomás, (UST). (2018). Fonoaudiología [Página institucional]. Recuperado 18 de marzo de 2018, de Universidad Santo Tomás website: http://www.ust.cl/carreras/fonoaudiologia/ 